\title{
Estudo da aplicaçáo dos componentes Atividade e Participaçáo da Checklist da CIF em pessoas com Esclerose Múltipla e sua relação com os Core Sets ${ }^{1}$
}

\author{
Rosé Colom Toldrá, Camila Landim do Nascimento \\ Departamento de Fisioterapia, Fonoaudiologia e Terapia Ocupacional, Universidade de São Paulo - USP, \\ São Paulo, SP, Brasil.
}

\begin{abstract}
Resumo: Introdução: O Core Set é um conjunto de categorias da Classificação Internacional de Funcionalidade, Incapacidade e Saúde - CIF, típicas e significativas, relacionadas à funcionalidade, utilizadas para determinadas condições de saúde. Objetivo: O estudo analisou a aplicação de categorias do componente Atividades e Participação baseadas na Checklist da CIF para esclerose múltipla (EM) e sua relação com o Core Set abrangente e o Core Set validado por terapeutas ocupacionais. Método: Pesquisa quantitativa descritiva, a partir de coleta e organização de dados de questionário baseado no componente Atividades e Participação Checklist da CIF - Versão 2.1. ${ }^{\text {- Formulário }}$ Clínico para a CIF, contendo 25 categorias pertencentes a seis domínios, e análise de sua relação com os Core Sets. Participaram 115 pessoas com EM remitente-recorrente, sem incapacidade até incapacidade moderada, acompanhadas no Instituto Central do Hospital das Clínicas da Faculdade de Medicina da Universidade de São Paulo. Foi criado banco de dados em Excel e feita análise descritiva das frequências. Resultados: A maioria dos participantes eram mulheres (85) com idade média de 32 anos e 1 mês $( \pm 7,4)$. Estudo amplia as evidências da aplicabilidade do Core Set abrangente com $88 \%$ (22) das categorias e do Core Set validado por terapeutas ocupacionais com $72 \%$ (18), em relação às categorias avaliadas. Dificuldades mais expressivas foram encontradas nas categorias d640 Realização das tarefas doméstica, d430 Levantar e carregar objetos e d850 Trabalho remunerado. Conclusão: Recomendam-se estudos sobre aplicação do Core Set por terapeutas ocupacionais para ampliar as evidências da prática clínica profissional e para a inclusão de categorias, conforme o contexto.
\end{abstract}

Palavras-chave: Classificação Internacional de Funcionalidade, Incapacidade e Saúde, Atividades Cotidianas, Participação Social, Esclerose Múltipla, Terapia Ocupacional.

\section{Application study of the components Activity and Participation of the ICF Checklist used in people with Multiple Sclerosis and its relation to the Core Sets}

\begin{abstract}
Introduction: The Core Set is a group of typical and significant functionality categories of the International Classification of Functioning, Disability and Health - ICF used for certain health conditions. Objective: The study reviewed the application of the component categories Activities and Participation based on the ICF Checklist for multiple sclerosis (MS) and its relationship with the comprehensive and validated Core Sets by occupational therapists. Method: Descriptive quantitative research from collection and organization of data questionnaire based on the components Activities and Participation of the ICF Checklist - Version 2.1 ${ }^{\text {a }}$. Clinical form for the ICF containing 25 categories belonging to 6 domains and analysis of its relationship with the Core Sets. Participated 115 people with relapsing-remitting MS without disability to moderate disability, treated at the Central Institute of Hospital Clinics of the Faculty of Medicine University of São Paulo. We created database in Excel and made descriptive
\end{abstract}

Autor para correspondência: Rosé Colom Toldrá, Departamento de Fisioterapia, Fonoaudiologia e Terapia Ocupacional, Universidade de São Paulo, Rua Cipotânea, 51, Cidade Universitária, CEP 05360-000, São Paulo, SP, Brasil, e-mail: rosetoldra@usp.br

Recebido em Out. 3, 2015; $1^{\text {a }}$ Revisão em Mar. 15, 2016; $2^{\text {a }}$ Revisão em Jun. 9, 2016; 3ª Revisão em Jul. 31, 2016 ; Aceito em Ago. 4 , 2016. 
analysis of frequencies. Results: Most participants were women (85), mean age 32 years and 1 month ( \pm 7.4$)$. Study broadens the evidence of the applicability of the Comprehensive Core Set with $88 \%$ (22) and Core Set validated by occupational therapists with $72 \%$ (18) compared evaluated categories. More significant difficulties were encountered in the categories d640 Doing housework, d430 Lifting and carrying objects and d850 Remunerative employment, which reflected on the performance of various activities and social participation. Conclusion: Studies on application of the Core Set validated by occupational therapists are recommended to extend the evidence of professional clinical practice and inclusion of categories depending on the context.

Keywords: International Classification of Functioning, Disability and Health, Activities of Daily Living, Social Participation, Multiple Sclerosis, Occupational Therapy.

\section{Introdução}

Estudos sobre Core Sets para Esclerose Múltipla (EM) se justificam pela incidência, pelas comorbidades e pelos acometimentos funcionais associados a essa condição de saúde, o que desafia profissionais da saúde no atendimento das necessidades dessas pessoas. A EM é uma doença crônica que se caracteriza pela abrangência e pela heterogeneidade de sintomas. Os mais comuns podem afetar seriamente a participação social das pessoas, como: fadiga, disfunção intestinal e de bexiga, prejuízos cognitivos e emocionais, depressão, espasticidade, distúrbios da marcha, problemas de visão, tontura e vertigem, tremores, distúrbios da fala e deglutiçáo, alteração de sensibilidade, espasticidade e dor, disfunção sexual e convulsóes (KARHULA et al., 2013; KESSELRING, 2004).

A doença acomete pessoas adultas jovens, principalmente na faixa etária dos 20 aos 40 anos, o que corresponde à fase da vida de maior produtividade dos indivíduos (TOLDRÁ, 2005; SANTOS; YOKOTA; DIAS, 2007), e pode acarretar restriçōes e interrupção das atividades e na participação social (KESSELRING et al., 2008).

A funcionalidade é cada vez mais valorizada para avaliar o impacto da EM sobre o indivíduo e a eficácia do tratamento e da reabilitação (KESSELRING et al., 2008). Essa condição de saúde interfere negativamente na capacidade de realização das atividades da vida diária (TOLDRÁ, 2005); no entanto, o conhecimento sobre as consequências nas atividades para as pessoas com EM é, ainda, restrito (LEXELL; IWARSSON; LEXELL, 2006), apesar da forte relação entre limitação no desempenho das atividades de vida diária e a restrição na participação das atividades sociais destas pessoas (CONRAD et al., 2012).

A Classificação Internacional de Funcionalidade, Incapacidade e Saúde (CIF) categoriza estados de saúde a partir da perspectiva do corpo, do indivíduo e da sociedade, provendo informaçóes sobre as repercussóes da doença e dos componentes de saúde que interferem sobre a funcionalidade, as atividades e a participação. A CIF fornece um vocabulário padrão e um quadro conceitual unificado, para descrição de estados de saúde de indivíduos e populaçôes (ORGANIZAÇÃO..., 2003a), e reflete o importante conceito de que a saúde é mais do que a ausência de doença (DARZINS; FONE; DARZINS, 2006; ORGANIZAÇÃO..., 2003a).

Desde a criação na década de 1980 , a CIF vem sendo cada vez mais utilizada em pesquisas e nas práticas em reabilitação em diferentes países (RUARO et al., 2012; STAMM et al., 2006). Seu quadro conceitual abrangente tem sido aplicado para descrição de estados de saúde e bem-estar das pessoas em literatura revisada por pares e relatórios oficiais. Entretanto, ainda está praticamente por ser incorporada na rotina clínica e de gestão na área de terapia ocupacional (DARZINS; FONE; DARZINS, 2006).

A CIF enfatiza uma importante mudança de terminologia e de paradigma, e sinaliza para um modelo biopsicossocial. É reconhecida como ferramenta útil aos terapeutas ocupacionais e suporte para comunicação entre diferentes profissōes (DARZINS; FONE; DARZINS, 2006). A Classificação é composta por 1.454 categorias, que descrevem aspectos da funcionalidade humana distribuídas entre componentes, como "Funções do corpo", "Estruturas do corpo", "Atividade", "Participaçẫo" e "Fatores Ambientais", portanto, consideravelmente abrangente. Visto que avaliar todas as categorias em todas as pessoas seria impraticável, foram desenvolvidos Core Sets da CIF, ou seja, categorias da CIF mais típicas e significativas relativas à funcionalidade para determinadas condiçóes de saúde (COENEN et al., 2011a; RIBERTO, 2011).

Especificamente para EM, em 2010, na Conferência de Consenso Internacional, foi criado o Core Set abrangente, que se destina a ambientes como o de reabilitação, com equipe multidisciplinar, em que se fazem necessários descrever e avaliar os sintomas da EM, e planejar a conduta terapêutica. Esse Core Set dispóe de 138 categorias, o que torna seu uso mais prático, para descrever os aspectos relevantes da 
funcionalidade e de saúde das pessoas com EM (COENEN et al., 2011a). Em 2012, o Core Set abrangente para EM foi validado por terapeutas ocupacionais (Core Set TO), sendo selecionadas 71 categorias e incluídas 11 novas, mais específicas para a área (CONRAD et al., 2012).

Para a Terapia Ocupacional, se destaca a aplicabilidade dos domínios e das categorias pertencentes ao componente Atividades e Participação, por estabelecer maior interlocução com a prática profissional (TOLDRÁ, 2003; DARZINS; FONE; DARZINS, 2006; LEXELL; IWARSSON; LEXELL, 2006). De acordo com a CIF, o componente Atividades e Participação tem a finalidade de indicar as várias açôes e áreas da vida, de maneira a cobrir a faixa completa de domínios, que denotam os aspectos da funcionalidade, tanto na perspectiva individual quanto social. Neste estudo, foram empregados os mesmos domínios tanto para atividades como para participação, ou seja, todos os domínios foram interpretados como funcionalidade individual (execução de uma tarefa ou ação por um indivíduo - atividade), bem como funcionalidade social (ato de se envolver em uma situação de vida - participação), conforme previsto pela CIF (ORGANIZAÇẢO..., 2003a).

Assim, o presente estudo se desenvolveu a partir da aplicação do componente Atividades e Participação baseada na Checklist da CIF (ORGANIZAÇÃO..., 2003b), para a avaliaçáo das principais áreas da vida afetadas pelas pessoas com EM. A criação dos Core Sets e a sua validação na perspectiva da Terapia Ocupacional durante a realização da pesquisa instigou estudar sua relaçáo com as categorias do componente Atividades e Participaçáo investigadas a partir da Checklist da CIF.

O estudo teve como objetivo analisar as principais categorias do componente Atividades e Participação, baseadas na Checklist da CIF (ORGANIZAÇÃO..., 2003b), aplicadas em pessoas com EM atendidas no Instituto Central do Hospital das Clínicas da Faculdade de Medicina da Universidade de Sáo Paulo (ICHC-FMUSP), para identificar as categorias relevantes para a avaliação da terapia ocupacional e sua relação com os Core Sets abrangente e o validado por terapeutas ocupacionais, para essa população.

\section{Método}

Trata-se de estudo quantitativo descritivo que investiga o uso de métodos para coleta e organização de dados, realizado a partir da aplicação da Check list da CIF em pessoas com EM e da análise de sua relação entre o Core Set abrangente e o Core Set validado na perspectiva da terapia ocupacional (Core Set TO). A pesquisa foi realizada com um grupo de 115 usuários com EM acompanhados pelo Ambulatório de Doenças Desmielinizantes da Disciplina de Neurologia no ICHC-FMUSP, conduzido de 2011 a 2012. O estudo foi aprovado pela Comissão de Ética para Análise de Projetos de Pesquisa (CAPPesq) da Diretoria Clínica do HC-FMUSP no 0163/11. Os participantes do estudo, após ciência e aceite em participar da pesquisa, assinaram o Termo de Consentimento Livre e Esclarecido.

O estudo foi desenvolvido a partir de amostra de conveniência estabelecida, conforme rotina do ambulatório, a partir de encaminhamentos dos dois primeiros usuários atendidos pelos neurologistas, que preenchiam os critérios da pesquisa e concordavam com a participação. No encaminhamento, constavam dados de diagnóstico e a avaliação da Escala Expandida do Estado de Incapacidade (EDSS). Essa escala quantifica as incapacidades em oito sistemas funcionais: piramidal, cerebelar, tronco cerebral, sensitivo, vesical, intestinal, visual e mental, com escore de 0-10 ('0', sem alteração neurológica; '5', incapacidade moderada, a qual afeta as atividades diárias, porém com autonomia para a sua realização, e '10', morte por EM) (CALLEGARO; LINO; MARCHIORI, 2009).

\subsection{Critérios de inclusão e exclusão}

Foram incluídos usuários que possuíam o diagnóstico da forma clínica remitente-recorrente (RR), na fase estável da doença (período mínimo de seis meses após um eventual surto); até grau de incapacidade medido pelo EDSS, com pontuação de 0-5 (nenhuma incapacidade até incapacidade moderada); idade de 18 a 45 anos; ambos os sexos, e sem limite do tempo de doença, devido aos sintomas serem muito variáveis ao longo do tempo. Adotados como critérios de exclusão os usuários com as formas secundariamente-progressiva, primariamente-progressiva, progressiva-recorrente da doença; idade acima de 45 anos; com surto havia menos de seis meses, usuários com outras doenças neurológicas associadas e/ou disfunção cognitiva que dificultasse responder ao questionário e com pontuaçáo de EDSS acima de 5.

\subsection{Instrumentos e coleta de dados}

A coleta de dados foi realizada após a consulta médica, em sala reservada, na forma de entrevista, com aplicaçáo de um questionário desenvolvido a partir de uma ferramenta prática denominada Checklist 
da CIF - Versão 2.1. a - Formulário Clínico para a CIF (ORGANIZAÇÃO..., 2003b). A Checklist é considerada uma das categorias principais da CIF, usada como uma ferramenta para extrair e registrar informação sobre a funcionalidade e a incapacidade de uma pessoa. O questionário foi aplicado com 25 das 48 categorias do componente Atividades e Participação, previstas na Checklist. Dos nove domínios do componente Atividades e Participação, foram estudadas categorias que pertencem a seis domínios, quais sejam: Mobilidade, Cuidado pessoal, Vida doméstica, Interaçóes interpessoais, Áreas principais da vida, e Vida comunitária, que representam 25 categorias estudadas das 35 categorias pertencentes a estes mesmos domínios.

O Core Set abrangente possui 138 categorias para $\mathrm{EM}$, sendo 53 referentes às categorias do componente Atividades e Participação. O Core Set TO possui 71 categorias do Core Set abrangente e foram incorporadas outras 11 categorias, distribuídas em Funçōes do Corpo (6), Atividades e Participação (2) e Fatores Ambientais (3), consideradas mais específicas da área, pois envolvem aspectos relacionados às intervenções de terapia ocupacional no cuidado às pessoas com EM (CONRAD et al., 2012). No total, 42 categorias pertencem ao componente Atividades e Participação. Assim, o estudo realizou a análise das 25 categorias pertencentes a seis domínios do componente Atividades e Participação da Checklist - CIF e sua relação com as 38 categorias do Core Set abrangente e as 34 categorias do Core Set TO, pertencentes a esses mesmos seis domínios.

\subsection{Procedimentos para análise dos dados}

Para agrupar e codificar as categorias dos seis domínios selecionados da Checklist - CIF com as categorias dos mesmos seis domínios do Core Set abrangente e do Core Set TO, foi criado um banco de dados em Excel. Em seguida, foi realizada a análise dos dados para identificação das categorias selecionadas da Checklist - CIF com o Core Set Abrangente e com o Core Set TO, e feito o cálculo da frequência, da porcentagem e do desvio padrão das respostas obtidas pelos participantes do estudo.

\section{Resultados}

Participaram do estudo, 85 mulheres $(73,9 \%)$ e 30 homens (26,1\%), sendo que $63(54,8 \%)$ dos participantes possuíam escolaridade no nível ensino superior completo e $65(56,6 \%)$ exerciam atividade laboral. A idade média era de 32 anos e 1 mês
$( \pm 7,4)$ e o tempo médio de doença era de 6 anos e 6 meses $( \pm 4,4)$. O EDSS máximo dos participantes foi igual a 5 , com média de $1,5( \pm 1,3)$, ou seja, sem incapacidade a incapacidade de moderada a leve.

As dificuldades apresentadas pelos participantes do estudo no componente Atividades e Participação desenvolvido a partir da Checklist CIF (ORGANIZAÇÃO..., 2003b) corresponderam aos seis domínios: Mobilidade; Cuidado pessoal; Vida doméstica; Relaçôes e interações interpessoais; Áreas principais da vida, e Vida comunitária, social e cívica, conforme dispostos na Tabela 1. Em todos os domínios, foram identificadas dificuldades, sendo que as maiores se apresentaram nos domínios Áreas principais da vida e Mobilidade, e a menor dificuldade, em Cuidado pessoal.

$\mathrm{Na}$ Tabela 2, estão relacionadas as 25 categorias da Checklist da CIF referentes aos seis domínios do formulário proposto; as 38 categorias estudadas (71\%) do Core Set Abrangente para EM do total de 53 categorias dos nove domínios que compóem o componente Atividades e Participação, e 34 categorias estudadas (80\%) do Core Set TO, do total de 42 categorias do mesmo componente.

Os resultados da pesquisa das 25 categorias do componente Atividades e Participação da Checklist da CIF mostraram que $22(88 \%)$ das categorias do Core Set Abrangente estão relacionadas às categorias estudadas pela Checklist da CIF. Quando se compara o total das categorias pertencentes aos seis domínios do mesmo componente, verificam-se 57\% (38) das categorias do Core Set Abrangente em relaçáo à Checklist da CIF. No Core Set TO, encontrou-se que $18(72 \%)$ das categorias estáo relacionadas às 25 categorias da Checklist da CIF. Ao se comparar o total das categorias que pertencem aos domínios estudados, observam-se $52 \%$ das categorias Core Set $\mathrm{TO}$ em relação às categorias estudadas na Checklist da CIF. Assim, com o estudo das 25 categorias da Checklist da CIF, obteve-se maior relaçáo com o Core Set Abrangente em comparação ao Core Set TO.

Das 25 categorias estudadas da Checklist da CIF, três não correspondem ao Core Set Abrangente: d740 - Relaçôes formais; d839 - Educação, outra especificada e não especificada, e d950 - Vida política e cidadania. Do Core Set TO, sete categorias não correspondem às categorias estudadas: $\mathrm{d} 740$ Relaçôes formais; d750 - Relações sociais informais; d760 - Relaçôes familiares; d770 - Relaçóes íntimas; d839 - Educação, outra especificada e não especificada; d870 - Autossuficiência econômica, e d950 - Vida política e cidadania. 
Tabela 1. Dificuldades apresentadas pelos participantes do estudo nos seis domínios do Componente Atividades e Participação a partir da Checklist da CIF. São Paulo, 2016.

\begin{tabular}{|c|c|c|c|}
\hline Mobilidade $(\mathrm{M}=38,1 \%)$ & & $\mathrm{n}^{\circ}$ & $\%$ \\
\hline d410 & Mudar a posição básica do corpo & 46 & $40 \%$ \\
\hline d430 & Levantar e carregar objetos & 49 & $42,6 \%$ \\
\hline d440 & Uso fino da mão & 48 & $41,7 \%$ \\
\hline d450 & Andar & 48 & $41,7 \%$ \\
\hline d470 & Utilização de transporte & 35 & $30,4 \%$ \\
\hline d475 & Dirigir & 37 & $32,7 \%$ \\
\hline \multicolumn{4}{|l|}{ Cuidado pessoal $(M=13,5 \%)$} \\
\hline d510 & Lavar-se & 12 & $10,4 \%$ \\
\hline d540 & Vestir-se & 11 & $9,5 \%$ \\
\hline d550 & Comer & 6 & $5,8 \%$ \\
\hline d570 & Cuidar da própria saúde & 33 & $28,6 \%$ \\
\hline \multicolumn{4}{|l|}{ Vida doméstica (M=29,5\%) } \\
\hline d6200 & Comprar & 26 & $22,6 \%$ \\
\hline d630 & Preparação de refeições & 27 & $23,4 \%$ \\
\hline d640 & Realização das tarefas domésticas & 58 & $50,4 \%$ \\
\hline d660 & Ajudar os outros & 25 & $21,7 \%$ \\
\hline \multicolumn{4}{|c|}{ Relações e interações interpessoais (M=28,6\%) } \\
\hline d740 & Relações formais & 27 & $23,4 \%$ \\
\hline d750 & Relações sociais informais & 36 & $31,3 \%$ \\
\hline d760 & Relações familiares & 34 & $29,5 \%$ \\
\hline d770 & Relações íntimas & 35 & $30,4 \%$ \\
\hline \multicolumn{4}{|c|}{ Áreas principais da vida $(\mathrm{M}=39,1 \%)$} \\
\hline d839 & Educação, outra especificada e não especificada & 43 & $37,3 \%$ \\
\hline d850 & Trabalho remunerado & 48 & $41,7 \%$ \\
\hline d870 & Autossuficiência econômica & 38 & $38,3 \%$ \\
\hline \multicolumn{4}{|c|}{ Vida comunitária, social e cívica $(\mathrm{M}=\mathbf{2 5 , 8 \%})$} \\
\hline d910 & Vida comunitária & 26 & $22,6 \%$ \\
\hline d920 & Recreação e lazer & 43 & $37,3 \%$ \\
\hline d930 & Religião e espiritualidade & 21 & $18,2 \%$ \\
\hline d950 & Vida política e cidadania & 29 & $25,2 \%$ \\
\hline
\end{tabular}

Tabela 2. Relação entre as categorias da Checklist da CIF selecionadas no estudo e o Core Set Abrangente e o Core Set TO para EM. São Paulo, 2016.

\begin{tabular}{|c|c|c|c|c|}
\hline \multirow{2}{*}{ Domínios/Categorias } & Checklist & \multirow{2}{*}{$\begin{array}{l}\text { Core Sets Abrangente } \\
\qquad 2^{\circ} \mathrm{N} .\end{array}$} & \multicolumn{2}{|c|}{ Core Sets TO } \\
\hline & $2^{\circ} \mathrm{N} .3^{\circ} \mathrm{N}$ & & $2^{\circ} \mathrm{N}$ & $3^{\circ} \mathrm{N}$ \\
\hline Mobilidade & & & & \\
\hline Mudar posição básica do corpo & $\mathrm{d} 410$ & $\mathrm{~d} 410$ & $\mathrm{~d} 410$ & \\
\hline Manter posição do corpo & & d4415 & & \\
\hline Permanecer deitado & & & & $\mathrm{d} 4150$ \\
\hline Permanecer sentado & & & & d44153 \\
\hline Permanecer em pé & & & & d44154 \\
\hline Transferir a própria posição & & $\mathrm{d} 420$ & $\mathrm{~d} 420$ & \\
\hline Levantar e carregar objetos & $\mathrm{d} 430$ & $\mathrm{~d} 430$ & $\mathrm{~d} 430$ & \\
\hline Uso fino da mão & $\mathrm{d} 440$ & d440 & $\mathrm{d} 440$ & \\
\hline Uso da mão e do braço & & d445 & $\mathrm{d} 445$ & \\
\hline Andar & $\mathrm{d} 450$ & $\mathrm{~d} 450$ & & \\
\hline Andar desviando-se de obstáculos & & & & $\mathrm{d} 4503$ \\
\hline Deslocar-se & & $\mathrm{d} 455$ & & \\
\hline Deslocar-se por diferentes locais & & $\mathrm{d} 460$ & $\mathrm{~d} 460$ & \\
\hline Deslocar-se utilizando algum tipo de equipamento & & $\mathrm{d} 465$ & d465 & \\
\hline Utilização de transporte & d470 & $\mathrm{d} 470$ & $\mathrm{~d} 470$ & \\
\hline Dirigir & $\mathrm{d} 475$ & $\mathrm{~d} 475$ & d475 & \\
\hline
\end{tabular}


Tabela 2. Continuação...

\begin{tabular}{|c|c|c|c|c|c|}
\hline \multirow{2}{*}{ Domínios/Categorias } & \multicolumn{2}{|c|}{ Checklist } & \multirow{2}{*}{$\begin{array}{c}\text { Core Sets Abrangente } \\
2^{\circ} \mathrm{N} .\end{array}$} & \multicolumn{2}{|c|}{ Core Sets TO } \\
\hline & $2^{\circ} \mathrm{N}$ & $3^{\circ} \mathrm{N}$ & & $2^{\circ} \mathbf{N}$ & $3^{\circ} \mathrm{N}$. \\
\hline \multicolumn{6}{|l|}{ Cuidado Pessoal } \\
\hline Lavar-se & $\mathrm{d} 510$ & & d5 510 & $\mathrm{~d} 510$ & \\
\hline Cuidado das partes do corpo & & & $\mathrm{d} 520$ & $\mathrm{~d} 520$ & \\
\hline Cuidados relacionados aos processos de excreção & & & $\mathrm{d} 530$ & $\mathrm{~d} 530$ & \\
\hline Vestir-se & $\mathrm{d} 540$ & & $\mathrm{~d} 540$ & $\mathrm{~d} 540$ & \\
\hline Comer & $\mathrm{d} 550$ & & $\mathrm{~d} 550$ & $\mathrm{~d} 550$ & \\
\hline Beber & & & $\mathrm{d} 560$ & $\mathrm{~d} 560$ & \\
\hline Cuidar da própria saúde & $\mathrm{d} 570$ & & $\mathrm{~d} 570$ & $\mathrm{~d} 570$ & \\
\hline \multicolumn{6}{|l|}{ Vida doméstica } \\
\hline Aquisição de bens e serviços & & & $\mathrm{d} 620$ & $\mathrm{~d} 620$ & \\
\hline Comprar & & d6200 & & & \\
\hline Preparação de refeições & $\mathrm{d} 630$ & & $\mathrm{~d} 630$ & $\mathrm{~d} 630$ & \\
\hline Realização das tarefas domésticas & $\mathrm{d} 640$ & & d640 & $\mathrm{d} 640$ & \\
\hline Cuidar dos objetos da casa & & & $\mathrm{d} 650$ & $\mathrm{~d} 650$ & \\
\hline Ajudar os outros & $\mathrm{d} 660$ & & d660 & d660 & \\
\hline \multicolumn{6}{|l|}{ Relações e interações interpessoais } \\
\hline Interações interpessoais básicas & & & d710 & d710 & \\
\hline Interações interpessoais complexas & & & d720 & & \\
\hline Interagir de acordo com regras sociais & & & & & d7203 \\
\hline Relações formais & $\mathrm{d} 740$ & & & & \\
\hline Relações sociais informais & $\mathrm{d} 750$ & & d750 & & \\
\hline Relações familiares & $\mathrm{d} 760$ & & $\mathrm{~d} 760$ & & \\
\hline Relações íntimas & d770 & & $\mathrm{d} 770$ & & \\
\hline \multicolumn{6}{|l|}{ Áreas principais da vida } \\
\hline Treinamento profissional & & & $\mathrm{d} 825$ & & \\
\hline Educação superior & & & $\mathrm{d} 830$ & & \\
\hline Educação, outra especificada e não especificada & d839 & & & & \\
\hline Conseguir, manter e sair de um emprego & & & $\mathrm{d} 845$ & $\mathrm{~d} 845$ & \\
\hline Trabalho remunerado & $\mathrm{d} 850$ & & $\mathrm{~d} 850$ & $\mathrm{~d} 850$ & \\
\hline Transações econômicas básicas & & & $\mathrm{d} 860$ & $\mathrm{~d} 860$ & \\
\hline Autossuficiência econômica & $\mathrm{d} 870$ & & $\mathrm{~d} 870$ & & \\
\hline \multicolumn{6}{|l|}{ Vida comunitária, social e cívica } \\
\hline Vida comunitária & d910 & & d910 & d910 & \\
\hline Recreação e lazer & d920 & & d920 & d920 & \\
\hline Religião e espiritualidade & d930 & & d930 & d930 & \\
\hline Vida política e cidadania & d950 & & & & \\
\hline Total de categorias & 25 & & 22 & 18 & \\
\hline
\end{tabular}

É dado que a pesquisa foi realizada com 25 categorias do componente Atividades e Participação da Checklist da CIF, envolvendo $41 \%$ das 53 categorias do Core Set abrangente pertencentes a esse componente; do Core Set TO, a pesquisa envolveu $42 \%$ das 42 categorias do componente Atividades e Participação.

No que se refere aos níveis das categorias, de acordo com a CIF, as categorias mais amplas - 2. ${ }^{\circ}$ nível - são definidas de forma a incluir implicitamente subcategorias mais detalhadas - $30^{\circ}$ e $40^{\circ}$ níveis (ORGANIZAÇÃO..., 2003a). Isso pode ser identificado no Core Set TO com cinco categorias de 3..$^{\circ}$ nível (quatro em Mobilidade e uma em Relaçóes e interaçóes interpessoais) e, da Cheklist da CIF, uma categoria de 3..$^{\circ}$ nível (Comprar).

\section{Discussão}

Este é o primeiro estudo no país que utiliza o Core Set abrangente da CIF e o Core Set TO para EM. Especificamente, foi realizada a verificação dos Core Sets com os resultados da aplicaçáo de 25 categorias do componente Atividades e Participação da Checklist da CIF (ORGANIZAÇÁO..., 2003b) em pessoas com EM atendidas no ICHC-FMUSP, dado que o estudo foi anterior ao desenvolvimento dos referidos 
Core Sets. Seus resultados ampliam as evidências da aplicabilidade do Core Set Abrangente e do Core Set $\mathrm{TO}$, tendo sido relacionadas à maioria das categorias do formulário estudado.

O uso da CIF, neste estudo, favoreceu o registro e a avaliaçáo dos resultados de forma padronizada, bem como contribuiu para um maior conhecimento sobre as consequências da doença nas atividades e na participação destas pessoas. Isso auxilia no estabelecimento de metas de intervenção em terapia ocupacional e facilita o diálogo com os demais profissionais (TOLDRÁ; SOUTO, 2014; CONRAD et al., 2012; DARZINS; FONE; DARZINS, 2006).

Verificou-se que, das 138 categorias do Core Set abrangente da CIF para EM, 53 são referentes a Atividades e Participação, o que demonstra a importância que o Core Set e a própria CIF atribuem a tal componente (COENEN et al., 2011a). Trata-se do componente que mais dialoga com o campo da Terapia Ocupacional (TOLDRÁ, 2003; DARZINS; FONE; DARZINS, 2006; LEXELL; IWARSSON; LEXELL, 2006) e constitui categorias cruciais para o trabalho clínico profissional (CONRAD et al., 2012).

Tal ênfase decorre da importância dada pela profissão na facilitação e na promoção de ocupaçôes significativas na vida diária das pessoas, referentes ao autocuidado, à produtividade e ao lazer, para atender as diferentes necessidades decorrentes das condiçôes de saúde e/ou sociais (LEXELL; IWARSSON; LEXELL, 2006). Isso vem reafirmar que o estudo e a aplicação das atividades dizem respeito tanto à competência quanto à identidade profissional dos terapeutas ocupacionais (STOFFEL; NICKEL, 2013).

Devido à composição da amostra, formada por uma população adulta jovem e com pouco comprometimento dos sistemas, pode-se justificar a maior relação encontrada com o Core Sets Abrangente. Este foi desenvolvido com a finalidade de descrever os principais aspectos relacionados à funcionalidade $\mathrm{e}$ à saúde de pessoas com EM, apresentando, portanto, caráter mais amplo do que o Core Set TO.

Em conformidade com as características da população, verificou-se que as maiores dificuldades apresentadas pelas pessoas com EM corresponderam aos domínios Áreas principais da vida e Mobilidade, em comparação com Cuidado pessoal, que apareceu com menor dificuldade, provavelmente devido às limitaçóes na realizaçáo das atividades de cunho pessoal surgirem quando há maior comprometimento nas funçôes e estruturas corporais.

O fato de a maioria da população do estudo ser do sexo feminino pode justificar o destaque das dificuldades enfrentadas na realizaçáo das tarefas domésticas, tendo sido citadas como de maiores dificuldades: d640 - Realização das tarefas domésticas (domínio Vida doméstica); d430 - Levantar e carregar objetos; d440 - Uso fino da mão; d450 - Andar, e d410 - Mudar a posição básica do corpo (domínio Mobilidade), presentes em ambos Core Sets.

Já as atividades que envolvem habilidades e papéis sociais, tais como d850 - Trabalho remunerado, d870 - Autossuficiência econômica, d470 - Utilização de transporte, que correspondem às atividades avançadas de vida diária e, portanto, mais complexas, podem ser indicativas de perdas funcionais futuras (DIAS et al., 2011). Chama a atenção a repercussão de tais dificuldades, tendo em vista que um pouco mais da metade da populaçáo do estudo exercia atividade laboral e era composta por adultos jovens.

No domínio Áreas principais da vida, destaca-se a categoria referente à escolaridade. A categoria d839 - Educaçáo, outra especificada e não especificada foi utilizada neste estudo no lugar de "d830 - Educação Superior”, presente no Core Set abrangente, por ser uma categoria mais geral e considerar diferentes níveis educacionais, o que melhor contempla a realidade brasileira. Ainda, nesse mesmo domínio, destaca-se a categoria d850 - Trabalho remunerado, que faz parte de ambos Core Sets. Tal categoria foi selecionada devido à totalidade da amostra se encontrar na faixa etária economicamente ativa, apontada como a segunda dificuldade mais expressiva. A incapacidade para o trabalho e a aposentadoria prematura ocorrem em adultos jovens e de média idade, com implicaçôes socioeconômicas importantes (CONRAD et al., 2012) e, portanto, refletem o significativo papel do trabalho na vida das pessoas e na sua participação social (COENEN et al., 2011a; TOLDRÁ, 2009).

Destaca-se a importância dada ao domínio Mobilidade pela população da amostra, especificamente à categoria $\mathrm{d} 450$ - Andar, presente em ambos Core Sets. Isso reforça a pertinência da categoria d 465 - Deslocar-se utilizando algum tipo de equipamento, nos próximos estudos, empregado no Core Set TO. A dificuldade de andar é um dos problemas que trazem maiores impactos na qualidade de vida da pessoa com EM (COENEN et al., 2011a) e remete à importância da avalição e da prescrição de equipamentos auxiliares para maior independência na marcha e na mobilidade. Como a aquisição de equipamentos adequados pode ser dispendiosa, utilizou-se, neste estudo, a categoria d870 - Autossuficiência econômica, pertencente ao domínio Áreas principais da vida, também presente no Core Set Abrangente.

Ainda no domínio Mobilidade, as três categorias d410 - Mudar posição básica do corpo, d4503 - Andar 
desviando-se de obstáculos e d460 - Deslocar-se por diferentes locais, empregadas no Core Set TO, se mostram importantes para uma avaliação mais apropriada das dificuldades para a realização de tais atividades, as quais podem comprometer o convívio e a participaçáo social das pessoas com EM. O domínio mobilidade apresenta o maior número de categorias nos dois Core Sets, além de ter sido a dificuldade mais referida no presente estudo, o que demonstra a sua importância mesmo se tratando de populaçáo com pouca incapacidade, conforme EDSS.

No domínio Vida doméstica, destaca-se a categoria d6200 - comprar (terceiro nível), utilizada neste estudo por ser uma atividade comum no dia a dia das pessoas e de mais fácil compreensão em nosso contexto cultural. Essa categoria foi usada em vez da denominação mais genérica d620 - Aquisição de bens e serviços, presente nos dois Core Sets.

Quanto ao domínio Relaçóes e interaçóes interpessoais, optou-se por categorias de relaçóes interpessoais particulares, para captar de forma mais específica as necessidades da população, em vez de categorias interpessoais gerais, como apresenta o Core Set TO. A categoria d740 - Relações formais, embora não incluída nos Core Sets, esteve presente no estudo preliminar para seleção do Core Set abrangente para EM (KHAN; PALLANT, 2007). A categoria d750 - Relações sociais informais, presente no Core Set Abrangente, foi avaliada neste estudo com maior dificuldade em comparação com as relaçôes formais, o que pode representar possíveis barreiras para a realização das atividades e da participaçáo social. De modo geral, o domínio Relaçóes e interaçóes interpessoais apresenta um grau importante de dificuldade, indicativo de que deve ser alvo da terapia ocupacional, pois as relaçóes e interaçóes sociais influenciam de sobremaneira no engajamento em atividades no contexto de vida.

No domínio Vida comunitária, social e cívica, destacam-se as categorias d910 - Vida comunitária e d920 - Recreação e lazer, presentes nos Core Sets e também neste estudo, dadas as oportunidades para a convivência social e para o envolvimento em outras situaçóes de vida que tais atividades propiciam. Também outros estudos sobre Core Sets para EM obtiveram, na categoria d920 - Recreação e lazer, expressividade equivalente com os resultados de nossa amostra, citada como um dos problemas mais importantes no desempenho ocupacional destas pessoas (KARHULA et al., 2013; CONRAD et al., 2012; COENEN et al., 2011a). Já a categoria d950 - Vida política e cidadania, embora não pertença aos Core Sets, foi incluída no estudo, devido ao seu papel na participação e na organização de pessoas com deficiência na conquista e na garantia de direitos, devido à desigualdade social do contexto brasileiro.

Indica-se que futuros estudos possam ser conduzidos em nosso país com a aplicação dos Core Sets. A pesquisa sugere que, nas próximas pesquisas com a aplicação do Core Set abrangente e Core Set TO, haja a inclusão das categorias: d839 - Educação, outra especificada e não especificada, e d870 - Autossuficiência econômica e, ainda, a categoria d950 - Vida política e cidadania, para a utilização do Core Set TO, devido às características do contexto histórico e social do nosso país.

Autores que pesquisam a aproximação da CIF e dos Core Sets com as problemáticas das pessoas com EM reforçam a relevância dos instrumentos de autoanálise, para a complementação de dados (KARHULA et al., 2013; COENEN et al., 2011b; HOLPER et al., 2010; PALTAMAA et al., 2008; KHAN; PALLANT, 2007). Assim, constatou-se no presente estudo, que os questionários aplicados na forma de entrevista ajudaram a identificar e a elucidar informaçóes importantes sobre a percepção dos entrevistados referentes à sua condição de funcionalidade.

Igualmente, não é esperado que todos os aspectos considerados relevantes para a terapia ocupacional possam ser traduzidos na linguagem da CIF. Dessa forma, é indicada a complementação com outros recursos (CONRAD et al., 2012), que possam colaborar na abrangência e na qualificação da prática clínica dos terapeutas ocupacionais. Do mesmo modo, as diferentes profissóes de saúde empregam seus próprios modelos de reabilitação e o uso da CIF e os Core Sets podem oferecer um quadro comum e útil, para a comunicação dos diferentes problemas de funcionalidade, bem como apoiar a comunicação das metas de reabilitação e a construção de um diagnóstico situacional e de intervençôes mais complexas e específicas, a partir das demandas das pessoas com EM (TOLDRÁ; SOUTO, 2014; COENEN et al., 2011a; RAUCH; STUCKI, 2008; DARZINS; FONE; DARZINS, 2006; RENTSCH et al., 2003).

Assim, verificou-se que a pesquisa potencializa o uso prático dos Core Sets. Como aplicabilidade, apresenta um panorama descritivo geral e avaliativo sobre as atividades e a participação, o que favorece a identificação das repercussôes das limitações no cotidiano de vida das pessoas com EM.

\section{Conclusão}

O estudo conduzido com pessoas adultas jovens e com pouca incapacidade aponta que a maioria das categorias do componente Atividades e Participação 
da Checklist da CIF do formulário proposto foi relacionada às categorias do Core Set abrangente para EM e do Core Set TO. Isso amplia as evidências desses instrumentos e assinala para a importância do desenvolvimento de estratégias para a aplicação do Core Set da CIF para a prática clínica da terapia ocupacional, a partir de diferentes perspectivas.

Como limitação da pesquisa, destaca-se o fato de a mesma ter sido realizada com parte dos domínios e categorias do componente Atividade e Participação baseado na Checklist da CIF. No entanto, como se trata de estudo realizado anteriormente ao desenvolvimento dos referidos Core Sets, os resultados da aplicaçáo da Checklist da CIF em pessoas com EM atendidas em um serviço de alta complexidade reforçam a utilidade dos Core Sets.

Recomenda-se que estudos de aplicação do Core Set TO possam ser conduzidos para maior aceitação e credibilidade de seu uso em nosso país e, quando necessário, incluir outras categorias de acordo com o contexto.

\section{Agradecimentos}

Ao Dr. Dagoberto Callegaro, responsável pelo Ambulatório de Doenças Desmielinizantes da Disciplina de Neurologia no ICHC-FMUSP e a equipe médica pelo apoio na coleta de dados do estudo.

\section{Referências}

CALLEGARO, D.; LINO, A. M. M.; MARCHIORI, P. E. Esclerose múltipla e outras doenças desmielinizantes do sistema nervoso central. In: MARTINS, M. A. et al. Clínica médica. Barueri: Manole, 2009. p. 449-478.

COENEN, M. et al. The development of ICF Core Sets for multiple sclerosis: results of the International Consensus Conference. Journal of Neurology, Berlin, v. 258, n. 8 , p. 1477-1488, 2011a. PMid:21373900. http:// dx.doi.org/10.1007/s00415-011-5963-7.

COENEN, M. et al. Functioning and disability in multiple sclerosis from the patient perspective. Chronic Illness, Thousand Oaks, v. 7, n. 4, p. 291-310, 2011b. PMid:21840913. http://dx.doi. org/10.1177/1742395311410613.

CONRAD, A. et al. Validation of the comprehensive ICF core set for multiple sclerosis from the perspective of occupational therapists. Scandinavian Journal of Occupational Therapy, Munich, v. 19, n. 6, p. 468-487, 2012. PMid:22428806. http://dx.doi.org/10.3109/11038128. 2012.665475 .

DARZINS, P.; FONE, S.; DARZINS, S. The International classification of functioning, Disability and Heal- th can help to structure and evaluate therapy. Australian Occupational Therapy Journal, Melbourne, v. 53, n. 2, p. 127-131, 2006. http://dx.doi.org/10.1111/j.14401630.2006.00580.x.

DIAS, E. G. et al. Caracterizaçáo das atividades avançadas. Revista de Terapia Ocupacional da Universidade de São Paulo, São Paulo, v. 22, n. 1, p. 45-51, 2011.

HOLPER, L. et al. Characterization of functioning in multiple sclerosis using the ICF. Journal of Neurology, Berlin, v. 257, n. 1, p. 103-113, 2010. PMid:19756827. http://dx.doi.org/10.1007/s00415-009-5282-4.

KARHULA, M. et al. The activities and participation categories of the ICF Core Sets for multiple sclerosis from the patient perspective. Disability and Rehabilitation, London, v. 35, n. 6, p. 492-497, 2013. PMid:22816381. http://dx.doi.org/10.3109/09638288 .2012.702845.

KESSELRING, J. et al. Developing the ICF Core Sets for multiple sclerosis to specify functioning. Multiple Sclerosis, London, v. 14, n. 2, p. 252-254, 2008. PMid:17986511. http://dx.doi. org/10.1177/1352458507082615.

KESSELRING, J. Neurorehabilitation in multiple sclerosis: what is the evidence-base? Journal of Neurolo$g y$, Berlin, v. 251, n. 2, p. 25-29, 2004. Suplemento 4. PMid:15378304.

KHAN, F.; PALLANT, J. F. Use of International Classification of Functioning, Disability and Health (ICF) to describe patientreported disability in multiple sclerosis and identification or relevant environmental factors. Journal of Rehabilitation Medicine, Stockholm, v. 39, n. 1, p. 63-70, 2007. PMid:17225040. http://dx.doi. org/10.2340/16501977-0002.

LEXELL, E. M.; IWARSSON, S.; LEXELL, J. The complexity of daily occupations in multiple sclerosis. Scandinavian Journal of Occupational Therapy, London, v. 13, n. 4, p. 241-248, 2006. PMid:17203674. http:// dx.doi.org/10.1080/11038120600840200.

ORGANIZAÇĀO MUNDIAL DA SAÚDE - OMS. CIF: Classificação Internacional de Funcionalidade, Incapacidade e Saúde. São Paulo: EDUSP, 2003a.

ORGANIZAÇÃO MUNDIAL DA SAÚDE - OMS. Checklist da CIF. Versão 2.1 a: formulário clínico para a Classificação Internacional de Funcionalidade, Incapacidade e Saúde. São Paulo: EDUSP, 2003b. Disponível em: <http://www.fsp.usp.br/cbcd/Material/CHECKLIST_DA_CIF.pdf >. Acesso em: 18 nov. 2010.

PALTAMAA, J. et al. Measuring deterioration in International Classification of Functioning domains of people with Multiple Sclerosis who are ambulatory. Physical Therapy, Alexandria, v. 88, n. 2, p. 176-190, 2008. PMid:18029390. http://dx.doi.org/10.2522/ ptj.20070064.

RAUCH, A. C. A.; STUCKI, G. How to apply the International Classification of Functioning, Disability and 
Health (ICF) for rehabilitation management in clinical practice. European Journal of Physical and Rehabilitation Medicine, Torino, v. 44, n. 3, p. 329-342, 2008. PMid:18762742.

RENTSCH, H. P. et al. The implementation of the "International Classification of Functioning, Disability and Health" (ICF) in daily practice of neurorehabilitation: an interdisciplinary project at the Kantonsspital of Lucerne. Disability and Rehabilitation, Stockholm, v. 25, n. 8, p. 411-421, 2003. PMid:12745951. http://dx.doi.or g/10.1080/0963828031000069717.

RIBERTO, M. Core sets da Classificação Internacional de Funcionalidade, Incapacidade e Saúde. Revista Brasileira de Enfermagem, Brasília, v. 64, n. 5, p. 938-946, 2011. http://dx.doi.org/10.1590/S003471672011000500021 .

RUARO, J. A. et al. Panorama e perfil da utilização da CIF no Brasil: uma década de história. Revista Brasileira de Fisioterapia, São Carlos, v. 16, n. 6, p. 1-9, 2012.

SANTOS, E.; YOKOTA, M.; DIAS, N. Esclerose múltipla: estudo de pacientes com a forma surto-remissão cadastrados na Secretaria de Estado da Saúde de Minas Gerais. Arquivos de Neuro-Psiquiatria, São Paulo, v. 65, n. 3b, p. 885-888, 2007. http://dx.doi.org/10.1590/ S0004-282X2007000500032.

STAMM, T. et al. Exploration of the link between conceptual occupational therapy models and the International Classification of Functioning, Disability and Health.
Australian Occupational Therapy Journal, Melbourne, v. 53, n. 1, p. 9-17, 2006.

STOFFEL, D. P.; NICKEL, R. A utilização da atividade como ferramenta no processo de intervenção do terapeuta ocupacional em reabilitação neurológica. Cadernos de Terapia Ocupacional da UFSCar, São Carlos, v. 21, n. 3, p. 617-622, 2013.

TOLDRÁ, R. C. Reflexôes acerca da Terapia Ocupacional na Atenção à Pessoa Portadora de Deficiência Física. In: PÁDUA, E. M. M.; MAGALHÂES, L.V. Terapia Ocupacional: teoria e prática. Campinas: Papirus, 2003. p. 49-60.

TOLDRÁ, R. C. Terapia ocupacional e o método self-healing: criando novas possibilidades de viver o corpo. In: PÁDUA, E. M. M.; MAGALHÂES, L. V. (Org.). Casos, memórias e vivências em Terapia Ocupacional. Campinas: Papirus, 2005. p. 97-114.

TOLDRÁ, R. C. Políticas afirmativas: opinião das pessoas com deficiência acerca da legislação de reserva de vagas no mercado de trabalho. Revista de Terapia Ocupacional da Universidade de São Paulo, São Paulo, v. 20, n. 2, p. 110-117, 2009.

TOLDRÁ, R. C.; SOUTO, A. C. F. Fatores contextuais da CIF como ferramentas de análise das implicaçóes da aquisição de deficiência física por pessoas atendidas pela Terapia Ocupacional. Cadernos de Terapia Ocupacional da UFSCar, São Carlos, v. 22, n. 2, p. 347-359, 2014. http://dx.doi.org/10.4322/cto.2014.061.

\section{Contribuição dos Autores}

Rosé Colom Toldra foi responsável pela concepção da pesquisa, coleta e elaboração do banco de dados. Participou da análise dos dados, redação do texto e revisão final do texto. Camila Landim do Nascimento realizou o levantamento e organização dos dados e estudo bibliográfico. Participou da análise dos dados e redação do texto. Todos os autores aprovaram a versão final do texto.

\section{Notas}

${ }^{1}$ Foram executados todos os procedimentos éticos para a realização da pesquisa, aprovada pela Comissão de Ética para Análise de Projetos de Pesquisa (CAPPesq) da Diretoria Clínica do Hospital das Clínicas da Faculdade de Medicina da Universidade de São Paulo (no 0163/11). 